\title{
Control of Immediate Early Gene Expression for Human Cytomegalovirus Reactivation
}

\author{
Donna Collins-McMillen ${ }^{1}$, Jeremy Kamil ${ }^{2}$, Nathaniel Moorman ${ }^{3}$ and Felicia Goodrum ${ }^{\text {** }}$ \\ ${ }^{1}$ Department of Immunobiology and BIO5 Institute, University of Arizona, Tucson, AZ, United States, ${ }^{2}$ Department of \\ Microbiology and Immunology, Louisiana State University Health Sciences Center - Shreveport, Shreveport, LA, \\ United States, ${ }^{3}$ Department of Microbiology and Immunology, Lineberger Comprehensive Cancer Center, University of North \\ Carolina at Chapel Hill, Chapel Hill, NC, United States
}

OPEN ACCESS

Edited by:

Emma Poole,

University of Cambridge,

United Kingdom

Reviewed by:

lan J. Groves,

University of Cambridge,

United Kingdom

Christine O'Connor,

Cleveland Clinic, United States

*Correspondence:

Felicia Goodrum

fgoodrum@arizona.edu

Specialty section:

This article was submitted to

Virus and Host,

a section of the journal

Frontiers in Cellular and Infection

Microbiology

Received: 20 June 2020

Accepted: 03 August 2020

Published: 17 September 2020

Citation:

Collins-McMillen D, Kamil J

Moorman N and Goodrum F (2020)

Control of Immediate Early Gene

Expression for Human

Cytomegalovirus Reactivation.

Front. Cell. Infect. Microbiol. 10:476.

doi: 10.3389/fcimb.2020.00476
Human cytomegalovirus ( $\mathrm{HCMV}$ ) is a beta herpesvirus that persists for life in the majority of the world's population. The persistence of HCMV in the human population is due to the exquisite ability of herpesviruses to establish a latent infection that evades elimination by the host immune response. How the virus moves into and out of the latent state has been an intense area of research focus and debate. The prevailing paradigm is that the major immediate early promoter (MIEP), which drives robust expression of the major immediate early (MIE) transactivators, is epigenetically silenced during the establishment of latency, and must be reactivated for the virus to exit latency and re-enter productive replication. While it is clear that the MIEP is silenced by the association of repressive chromatin remodeling factors and histone marks, the mechanisms by which HCMV de-represses MIE gene expression for reactivation are less well understood. We have identified alternative promoter elements within the MIE locus that drive a second or delayed phase of MIE gene expression during productive infection. In the context of reactivation in THP-1 macrophages and primary CD34+ human progenitor cells, MIE transcripts are predominantly derived from initiation at these alternative promoters. Here we review the mechanisms by which alternative viral promoters might tailor the control of viral gene expression and the corresponding pattern of infection to specific cell types. Alternative promoter control of the HCMV MIE locus increases versatility in the system and allows the virus to tightly repress viral gene expression for latency but retain the ability to sense and respond to cell type-specific host cues for reactivation of replication.

Keywords: cytomegalovirus, alternative promoter usage, herpesvirus, latency, reactivation, major immediate early (MIE) promoter

Human cytomegalovirus (HCMV) is one of nine herpesviruses infecting humans. Primary HCMV infection is typically asymptomatic. Like all herpesviruses, HCMV evades immune clearance and establishes a life-long latent persistence (Goodrum, 2016). The latent persistence is marked by sporadic but likely frequent and typically subclinical reactivation events. Reactivation results in serious disease risk in those undergoing immune suppressive therapy associated with solid organ or stem cell transplantation or intensive chemotherapy associated with cancer treatment. In healthy adults, the cost or benefit of asymptomatic HCMV carriage is not well understood. HCMV seropositivity is associated with increased risk for cardiovascular disease and immune 
dysfunction in the aged (Streblow et al., 2008; NikolichZugich et al., 2017). However, there is also evidence that HCMV persistence may boost immune responses to infection or vaccination (Furman et al., 2015).

HCMV infects a diverse array of human cell types. While the biology of HCMV infection has been most thoroughly characterized in the context of robust productive replication in fibroblasts, other patterns of infection are unique to other cell types. For example, replication in epithelial cells and endothelial cells results in a chronic or low-level smoldering infection (Jarvis and Nelson, 2002, 2007; Adler and Sinzger, 2009). The factors driving these different infection outcomes are currently unclear, as the differences in viral gene expression or the manipulation of host pathways associated with infection in these cell types have not been systematically dissected relative to infection in fibroblasts. The latent pattern of HCMV infection is thought to be restricted to hematopoietic progenitors or cells of the myeloid lineage, and has been most thoroughly characterized in CD34+ hematopoietic progenitors (HPCs) (Goodrum, 2016). The ability of HCMV to infect and establish unique patterns of infection in a variety of cell types is indicative of the exceptional complexity the virus has attained through its co-evolution with its host, and is undoubtedly key to its lifelong persistence. Cell type-specific regulation of viral patterns of infection is a significant gap in our understanding of HCMV persistence. In this review, we focus on the regulation of re-expression of major immediate early genes following reactivation.

During latency, the viral genome is maintained in the absence of virus replication (Goodrum, 2016). Viral gene expression associated with latency has been challenging to define due to the heterogeneity of hematopoietic cell subpopulations. Conventionally, herpesvirus latency has been thought to be a silent state where viral gene expression is restricted to a limited number of genes-the so-called latency genes. However, recent transcriptomics studies across the herpesvirus field, suggest a much more active and dynamic state of latency (CollinsMcMillen and Goodrum, 2017; Singh and Tscharke, 2020). In HCMV infection of hematopoietic cells, the viral genes are broadly expressed, but at exceptionally low levels (Cheng et al., 2017; Shnayder et al., 2018). These findings have complicated defining distinct latent patterns of gene expression or the use of IE gene expression alone as an indicator of reactivation. Nevertheless, many studies have used the relative levels of immediate early or replicative genes (e.g., IE-86kDa, IE2-72kDa, UL135) to latency genes (e.g., UL138, LUNA) to define the latent state where the ratio of replicative to latent genes is greater in replicative states than in latent states (Kim et al., 2017; Shnayder et al., 2018; Buehler et al., 2019; Krishna et al., 2019).

In latency, viral gene expression is restricted by chromatinization and epigenetic regulation of the viral chromosome (Murphy et al., 2002; Reeves et al., 2005; Abraham and Kulesza, 2013; Albright and Kalejta, 2016). Studies of chromatin changes associated with latency and reactivation in HCMV have primarily focused on the major immediate early promoter (MIEP) (Woodhall et al., 2006; Groves et al., 2009). The MIEP is a complex promoter, consisting of an enhancer ( -520 to -65 nucleotides, $\mathrm{nt})$, a unique region $(-780$ to -610 $\mathrm{nt})$, and a modulator $(-1145$ to $-750 \mathrm{nt})$ in addition to the core promoter $(-65$ to $+3 \mathrm{nt})$. While the promoter is sufficient for transcription of IE genes (Thomsen et al., 1984), the enhancer element strongly enhances transcription (Boshart et al., 1985). The modulator has context dependent roles, repressive to MIEP activity in undifferentiated cells, but actively drives the MIEP in permissive fibroblasts (Nelson et al., 1987; Lubon et al., 1989; Shelbourn et al., 1989; Huang et al., 1996). The MIEP drives robust expression of the major immediate early genes, UL122 and UL123, encoding a number of major immediate early proteins, most notably the IE1-72kDa and IE2-86kDa major transactivators. The MIEP is initially transactivated by host transcription factors and gives rise to a common RNA precursor that is alternatively spliced (Stenberg et al., 1985; Liu and Stinski, 1992; Collins-McMillen et al., 2018). IE1-72kDa is encoded by exons 1-4 and IE2-86kDa is produced by an exon skipping event and is encoded by exons 1-3 and 5. Preferential splicing results in early accumulation of IE1, which switches at later times to favor IE2 (Sanchez et al., 2004; Oduro et al., 2012; Lin et al., 2017).

Chromatinization and silencing of the viral genome has been broadly studied across the herpesvirus family and plays a uniformly critical role in silencing viral gene expression for latency (Reeves and Sinclair, 2013; Lieberman, 2015; Cliffe and Wilson, 2017; Hopcraft et al., 2018). In HSV-1 infection, the genome is chromatinized as facultative heterochromatin, which is marked by methylation of lysine 27 on histone 3 (H3K27me3). Facultative chromatin is critical to the regulation of gene expression and is thought to be readily converted to euchromatin to activate gene expression (Trojer and Reinberg, 2007). Therefore, facultative heterochromatin may poise the herpesvirus genomes to readily respond to host cues for reactivation and may contribute to low level or sporadic gene expression during latency (Cliffe et al., 2009, 2013). Heterochromatinization of the whole HCMV genome remains to be fully characterized. Histone deacetylase activity reduces viral gene expression in models for latent HCMV infection (Wright et al., 2005; Saffert et al., 2010), corresponding to increased association of heterochromatin protein 1 (HP1) (Murphy et al., 2002) and the co-repressor KAP1 (Rauwel et al., 2015) with the MIE locus to contribute to silencing the viral genome. KAP1 initiates the formation of heterochromatin by recruiting HP1a and SETDB1 to trigger H3K9 methylation. Further, the polycomb repressive complex 2 (PRC2) increases H3K27me3 marks on the HCMV genome in undifferentiated cell line models of latency (Abraham and Kulesza, 2013). When PRC2 is inhibited, H3K27me3 marks associated with the viral genome decrease and viral gene expression increases, indicative of a role for PRC2 in chromatinization and repression of the viral genome for the establishment of latency.

Many host factors with the potential to suppress viral gene expression are components of PML or ND10 bodies, including PML, Daxx, and Sp100 (Saffert and Kalejta, 2006, 2007; Woodhall et al., 2006). While knockdown of Daxx increases IE gene expression in THP-1 or CD34+ cells infected with the AD169 laboratory strain (Saffert and Kalejta, 2007), it fails to rescue IE expression in cells infected with a low-passage strain (Saffert et al., 2010) or in undifferentiated THP-1 (Wagenknecht et al., 2015) or NTera2 (Groves and Sinclair, 2007) cells. These findings collectively, and not surprisingly, indicate that the repression of 
gene expression for latency is multifactorial and complex. Despite the ability of the pp71 tegument protein to stimulate viral gene expression by antagonizing Daxx, pp71 fails to traffic to the nucleus and degrade Daxx in cells that support latency (Saffert et al., 2010), a restriction that is overcome by higher multiplicities of infection or virions with high levels of pp71 (Woodhall et al., 2006; Chaturvedi et al., 2020).

Type 1 interferon (IFN) response upregulates PML-associated host factors and reversibly blocks IE gene expression to drive latency in MCMV-infected endothelial cells (Dag et al., 2014). In the context of HCMV infection, US28-mediated downregulation of IFN responsive genes is required for latency and IFI16 stimulates IE gene expression via NFkB for reactivation (Elder et al., 2019). In HSV-1 infection, IFI16 restricts HSV-1 gene expression and is targeted by ICP0 for destruction to stimulate gene expression (Orzalli et al., 2012; Merkl and Knipe, 2019). These studies suggest a complex role for the IFN response in contributing to latency, which is an area of important ongoing research in the field.

SAMHD1 is a restriction factor that depletes the pool of available dNTPs to suppress DNA polymerase processivity and has recently been shown to restrict MIE gene expression and replication by impeding $\mathrm{NFkB}$ activation in myeloid cells (Kim et al., 2019). In primary human macrophages, HCMV counteracts this restriction by inducing phosphorylation of SAMHD1 (T592) via the activity of the viral protein kinase, UL97, or activation of CDKs (Businger et al., 2019). HCMV infection also downregulates SAMHD1 transcript and protein levels. This viral strategy is conserved in MCMV where the M97 kinase phosphorylates SAMHD1 (Deutschmann et al., 2019). These findings indicate a role for SAMHD1 in suppressing replication for latency and SAMHD1 inactivation is required for reactivation. Further, the phosphatase CDC25B and CDK1 are repressive to viral gene expression, and inhibition of CDK1 stimulates viral gene expression in the Kasumi-3 model of latency (Pan et al., 2016). These studies collectively illustrate the complex and multi-layered approach to silencing viral gene expression for the establishment of latency.

While much remains to be understood about the signaling events and coordination of repressive activities to repress viral gene expression for latency, much less is known about how these layers of control are unraveled for reactivation. Reactivation necessarily depends on counteracting the strong, layered epigenetic silencing associated with latency. The reactivation of CMV from latency has been intimately linked to changes in signaling and hematopoietic differentiation (Soderberg-Naucler et al., 1997, 2001; Reeves and Compton, 2011; Huang et al., 2012; Kew et al., 2014; Buehler et al., 2016, 2019; Crawford et al., 2018; Forte et al., 2018; Mikell et al., 2019). Differentiation of latently infected cells along the myeloid lineage results in the reexpression of viral genes and reactivation (Ibanez et al., 1991; Taylor-Wiedeman et al., 1994; Soderberg-Naucler et al., 1997, 2001; Reeves and Sinclair, 2013). The chromatin remodeling associated with HCMV reactivation during hematopoietic differentiation is incompletely defined compared to the silencing events that drive latency (Reeves et al., 2005; Dupont et al., 2019). One driver of chromatin remodeling during reactivation is the FACT (facilitates chromatin transcription) complex, which functions to reposition histones and to increase accessibility to RNA polymerase. FACT is bound to the MIE locus both during latency and following reactivation events in Kasumi 3 cells, and is important for the re-expression of IE genes (O'Connor et al., 2016). Other viral proteins, including UL7, UL135 and specific isoforms of UL136, have also been shown to be required for reactivation (Umashankar et al., 2014; Caviness et al., 2016; Crawford et al., 2018; Rak et al., 2018; Mikell et al., 2019). HCMVcoded miRNAs have also emerged as important regulators of host signaling pathways that contribute to reactivation (Mikell et al., 2019).

Reactivation is associated with changes in the levels or binding of host transcriptional activators to the MIE region that stimulates reactivation (Bain et al., 2003; Liu et al., 2008; Kew et al., 2014; O’Connor et al., 2016; Krishna et al., 2019) and contains a high density of binding sites for host transcription factors important in inflammation and differentiation (CollinsMcMillen et al., 2018). As an example, the CREB host transcription factor binds to CRE sites within the MIE enhancer and also recruits mitogen and stress activated kinases that initiate chromatin remodeling, resulting in increased IE gene expression in infected monocytes as they differentiate into mature dendritic cells (Kew et al., 2014). Studies in NT2 cells differentiated with phorbol ester treatment have defined a cooperative role for CREB and an additional host transcription factor NF- $\mathrm{B}$ in driving reexpression of the IE genes (Liu et al., 2010; Yuan et al., 2015). However, most, if not all, studies assessing MIE activity following reactivation demonstrate only the accumulation of IE1 and IE2 transcripts or protein, and MIEP activity is presumed without being definitively demonstrated.

Because the MIEP is strongly repressed during latency and chromatin modifications in the MIE region correlate with cellular differentiation and the permissive state, it has been presumed that de-repression of the MIEP is a prerequisite to re-expression of IE genes upon reactivation (Figure 1A). Recent studies identified MIE transcripts encoding IE1-72kDa and IE2-86kDa proteins that have unique $5^{\prime}$ ends compared to those expressed from the MIEP (Arend et al., 2016). Two alternative transcriptional start sites were mapped within intron A of the MIE locus and correspond to two putative promoters, intronic promoters 1 and 2 (iP1 and iP2). The first initiates 350 nucleotides downstream $/ 3^{\prime}$ of the canonical exon 1 splice donor site and has an untranslated region of 378 nucleotides. The second initiates 54 nucleotides upstream $/ 5^{\prime}$ of the canonical exon 2 spice acceptor site and has an untranslated region of 70 nucleotides. Both of these transcripts lack the MIE exon 1. However, as exon 1 is non-coding, these transcripts still support synthesis of full-length IE1 and IE2 proteins. Intriguingly, these transcripts accumulate late in infection following the onset of viral genome synthesis in fibroblasts and their accumulation can be blocked with inhibitors of viral genome synthesis (Arend et al., 2016). The expression of these alternative MIE transcripts correlates with the second phase of IE2 protein accumulation that occurs late in infection of fibroblasts (Arend et al., 2016; CollinsMcMillen et al., 2019) and is consistent with continued IE gene 


\section{Latency}

A Paradigm



B Revised Paradigm

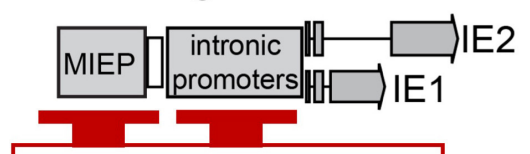

Signaling

Host epigenetic/

Transcription repressors

Viral factors?

\section{Reactivation}
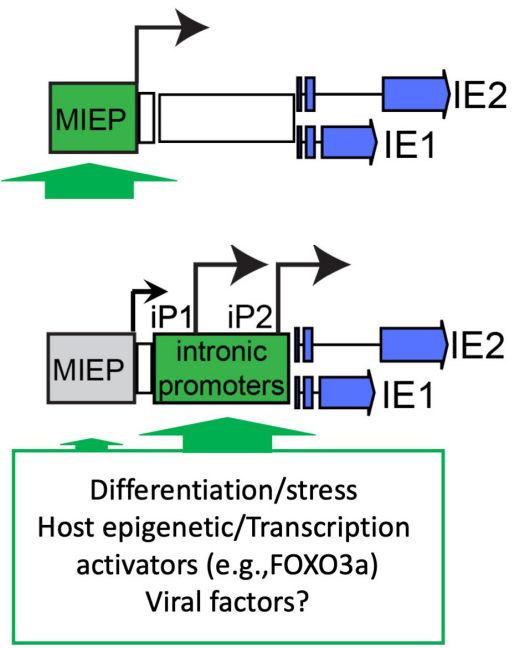

FIGURE 1 | Paradigms of HCMV latency and reactivation. (A) Silencing of the MIEP is required for HCMV latency, and it has long been thought that reactivation depends on de-repression of the MIEP for re-expression of IE genes. (B) Our recent work sheds new light on the control of MIE gene re-expression in THP-1 cell line and $\mathrm{CD}_{3} 4^{+}$primary cell models of latency and reactivation. Specifically, it defines alternative promoters within intron A of the MIE locus that give rise to full length IE1 and IE2 proteins. The intronic promoters must also be silenced for latency, similar to the MIEP. An additional post-transcriptional/translational regulation is likely involved, as low levels of iP2-derived transcripts are present during latency. Strikingly, reactivation stimuli in both models induce re-expression of IE1 and IE2 predominantly from the intronic promoters and to a much lesser extent, if at all, from the MIEP. The activation of the intronic promoters is regulated, at least in part, by the host transcription factor, FOXO3a, associated with hematopoietic differentiation. Other viral and cellular factors likely contribute to regulation of the MIE intronic promoters.

expression despite cis repression of the MIEP late in infection (Pizzorno and Hayward, 1990; Cherrington et al., 1991; Liu et al., 1991; Macias and Stinski, 1993; Reeves et al., 2006; Teng et al., 2012). While the promoter sequences present in intron A (herein referred to collectively as intronic promoters) await fine mapping, they are capable of driving IE gene expression outside the context of infection and from constructs containing the entire MIE locus (enhancer to $3^{\prime}$ UTR) where the MIEP core promoter $(-94$ to +64 relative to the transcription start site) has been deleted (Arend et al., 2016; Hale et al., 2020). These findings suggest that while the MIEP drives robust IE gene expression early in infection, additional promoters drive sustained IE gene expression late in infection and are presumably subject to distinct regulation. It should also be noted that CTCF binds within Intron A ( +834 to 852 , between iP1 and iP2) to negatively regulate MIE gene expression (Martinez et al., 2014). Disruption of CTCF binding increased MIE gene expression, but conveyed only a modest replicative advantage in fibroblasts and has not been analyzed in other contexts. The deletion of the intronic promoters also disrupts the CTCF binding site within intron A.

While MIE gene expression from the intronic promoters is not required for virus replication in fibroblasts, the presence of these alternative MIE promoters offers exciting possibilities with regard to the control of MIE gene expression in the context of latency and reactivation. This is particularly true in light of findings that MIEP activity is weak in cells that support HCMV latency, including $\mathrm{CD} 34^{+}$HPCs and $\mathrm{CD} 14^{+}$ cells (Shelbourn et al., 1989; Sinclair et al., 1992), which has precluded use of the MIEP to drive transgene expression for gene therapy. Using the THP-1 model for latency, we have shown that a transient burst of IE gene expression occurs immediately following infection but is gradually silenced. Following the stimulation of reactivation with phorbol ester, re-expression of MIE genes is induced. The MIE transcripts expressed in this model originate predominantly from the intronic promoters, not the MIEP (Collins-McMillen et al., 2019). Following reactivation of HCMV in CD34+ HPCs infected in vitro, MIE transcripts are also predominantly derived from the intronic promoters, and mutant viruses wherein the intronic promoters are disrupted reactivate poorly following stimulation in infected THP- 1 or $\mathrm{CD} 34^{+}$HPCs.

These findings suggest a model whereby the MIEP is silenced upon the establishment of latency and re-expression of MIE genes and reactivation of the replicative cycle requires switching to the intronic promoters to drive MIE gene expression (Figure 1B). At least in the contexts we have tested to date, no substantial re-expression from the MIEP has been detected. However, these findings do not preclude the possibility that robust reactivation stimuli lead to re-expression from the MIEP to drive virus replication. In a recent study by Mason and colleagues, transcription from both the MIEP and iP2 was observed in the THP-1 model and in CD14 ${ }^{+}$monocytes treated with phorbol ester (Mason et al., 2020). Further, in dendritic cells derived from either $\mathrm{CD}_{3} 4^{+}$or $\mathrm{CD} 14^{+}$cells and in $\mathrm{CD} 14^{+}$-derived macrophages stimulated with IL-1b and M-CSF, MIE transcripts 
were predominantly derived from the MIEP. These findings raise the possibility that regulation of the MIE locus, including the usage of various transcription factor binding sites and distinct promoters, is cell type-specific. This level of complexity would allow the virus to respond to multiple reactivation stimuli using different signaling pathways. In support, the transcription factors CREB and NF- $\mathrm{B}$, which bind the MIE enhancer region upstream of the MIEP transcription start site, were shown to play a cooperative role in re-initiating MIE gene expression in NT2 cells treated with phorbol ester (Liu et al., 2010; Yuan et al., 2015), whereas deletion of the CREB, but not the NF- $\kappa B$, binding sites resulted in decreased MIE expression in dendritic cells (Kew et al., 2014). It is also possible that host transcription factor binding sites in the MIE enhancer contribute to changes in 3D chromatin structure and the activity of other regions of the MIE locus, such as the alternative promoters, to drive MIE reexpression. In fact, the activator protein-1 (AP-1) transcription factor binding site in the MIE enhancer has been recently shown to stimulate activation of a MIE intronic promoter, in addition to the MIEP and distal promoter (Krishna et al., 2020). Regardless, our studies indicate an important role for the intronic promoters in re-expression of MIE genes and in reactivation from latency. This promoter switching mechanism provides the versatility necessary to strongly repress the MIE gene expression for latency, while preserving responsiveness to specific host cues for reactivation.

Providing insight into the regulation of the intronic promoters, intron A sequences contain consensus binding sites for the forkhead family transcription factors. The MIE intronic promoters can be activated by both FOXO1 and FOXO3a members; however, FOXO3a appears to be the transcription factor critical for reactivation (Hale et al., 2020). Mutating FOXO binding sites in the MIE intronic promoters diminishes reactivation of HCMV from latency in THP-1 cells or CD34 ${ }^{+}$ HPCs, demonstrating a role for FOXO transcription factors in reactivation. This is intriguing, as FOXO3a localizes to the nucleus or is induced in myeloid progenitor cells in response to cellular stresses and differentiation (Paik et al., 2007; Tothova and Gilliland, 2007; Yalcin et al., 2008; Liang et al., 2016). Further, FOXO3a expression is induced by differentiation stimuli in THP-1 cells (Hale et al., 2020). These findings suggest a mechanism by which the virus can sense and respond to host cues for reactivation through FOXO3a activation of the MIE intronic promoters.

Transcripts with alternative $5^{\prime}$ ends may be driven by promiscuous initiation of transcription or alternative promoter usage. Either mechanism serves to provide additional, contextdependent regulation of gene expression or to expand the limited coding potential within a viral genome. The $\sim 240$ base pair HCMV genome is estimated to encode $>165$ ORFs (Davison et al., 2003; Murphy et al., 2003a,b) and as many as 604 non-canonical ORFs (Stern-Ginossar et al., 2012). Over 7,000 transcriptional start sites are detected during lytic replication, a number far exceeding the number of ORFs that have been predicted. RNA polymerase II initiation of HCMV transcription is pervasive (Stern-Ginossar et al., 2012; Parida et al., 2019), with transcriptional start sites clustering within a 20-basepair interval. This indicates that HCMV may have regions to initiate transcription, rather than precise transcriptional start sites (Parida et al., 2019). Some of these start sites may produce alternative $5^{\prime}$ UTRs for differential regulation of translation, affecting ribosome initiation and progression (Mizrahi et al., 2018). Indeed, the $5^{\prime}$ UTRs of MIE transcripts play important roles in regulating the efficiency of translation and enhance translation in the context of infection (Arend et al., 2018).

UL136 is encoded on a polycistronic locus spanning UL133UL138 that encodes multiple proteins important to viral latency and reactivation. UL136 protein is not synthesized from longer transcripts encoding UL133 or UL135 (Grainger et al., 2010; Umashankar et al., 2011). Rather, UL136 is synthesized from multiple viral transcripts with unique $5^{\prime}$ ends (Caviness et al., 2014). These transcripts accumulate robustly in the late phase and depend on viral genome synthesis, whereas UL133, UL135, and UL138 are expressed with early kinetics. Unlike the MIE transcripts, which have identical coding sequences resulting in synthesis of either IE1-72kDa or IE2-86kDa whether derived from the MIEP or from the intronic promoters, the nested UL136 transcripts have unique $5^{\prime}$ ends that give rise to a series of unique proteins, each with a successive truncation amino terminal end compared to the full-length UL136 protein (Caviness et al., 2014). The UL136 promoter has not been mapped and it is not yet clear if UL136 transcripts arise from multiple promoters or initiation of transcript or how they might be differentially regulated in different contexts of infection. However, mutant viruses lacking single or combinations of the UL136 variants have revealed important roles for each in either stimulating or suppressing virus replication in endothelial cells and for the establishment of latency or reactivation from latency in CD34+ HPCs and humanized mice (Caviness et al., 2016).

Alternative promoters play key roles in tailoring gene expression for cell lineages, tissue types, developmental stages, stress and differentiation (Davuluri et al., 2008). Although not well characterized for HCMV, other herpesvirus family members also use alternative promoters for context-dependent regulation of gene expression. The IE transactivator, ICP27, of Marek's disease virus is expressed by different promoters in both the lytic and latent contexts of infection (Strassheim et al., 2016). Also, ORF50 of Kaposi's Sarcoma-associated herpesvirus (KSHV) is expressed from a series of transcripts driven by four distinct promoters, giving rise to six differentially spliced ORF50 transcripts, which results in multiple isoforms of the major transactivator RTA (Wakeman et al., 2017). RTA isoforms regulate the KSHV cascade of gene expression, each driving distinct transactivation programs for reactivation, and exhibit variable activity in different cell types. Finally, Epstein-Barr Virus (EBV) uses promoter switching for its distinct transcriptional programs for latency, and specifically the expression of the EBNA-1 latency protein (Woisetschlaeger et al., 1990; Schaefer et al., 1995; Nonkwelo et al., 1996; Tempera et al., 2011). The significance of these promoter switching events to the viral gene expression program, and how they are regulated remains to be fully understood. However, from these limited examples, and now the demonstration of promoter switching events in 
regulation of MIE gene expression for HCMV reactivation, it appears that promoter switching is a prominent mechanism for regulating gene expression for latency and reactivation.

\section{AUTHOR CONTRIBUTIONS}

FG wrote manuscript. DC-M and NM contributed data supporting manuscript and revised manuscript. JK contributed data supporting manuscript, revised manuscript, and

\section{REFERENCES}

Abraham, C. G., and Kulesza, C. A. (2013). Polycomb repressive complex 2 silences human cytomegalovirus transcription in quiescent infection models. J. Virol. 87, 13193-13205. doi: 10.1128/JVI.02420-13

Adler, B., and Sinzger, C. (2009). Endothelial cells in human cytomegalovirus infection: one host cell out of many or a crucial target for virus spread? Thromb. Haemost. 102, 1057-1063. doi: 10.1160/TH09-04-0213

Albright, E. R., and Kalejta, R. F. (2016). Canonical and variant forms of histone $\mathrm{H} 3$ are deposited onto the human cytomegalovirus genome during lytic and latent infections. J. Virol. 90, 10309-10320. doi: 10.1128/JVI.01220-16

Arend, K. C., Lenarcic, E. M., and Moorman, N. J. (2018). The 5' untranslated region of the major immediate early mRNA is necessary for efficient human cytomegalovirus replication. J. Virol 92, e02128-17. doi: 10.1128/JVI. 02128-17

Arend, K. C., Ziehr, B., Vincent, H. A., and Moorman, N. J. (2016). Multiple transcripts encode full-length human cytomegalovirus IE1 and IE2 proteins during lytic infection. J. Virol. 90, 8855-8865. doi: 10.1128/JVI.00741-16

Bain, M., Mendelson, M., and Sinclair, J. (2003). Ets-2 repressor factor (ERF) mediates repression of the human cytomegalovirus major immediate-early promoter in undifferentiated non-permissive cells. J. Gen. Virol. 84 (Pt. 1), 41-49. doi: 10.1099/vir.0.18633-0

Boshart, M., Weber, F., Jahn, G., Dorsch-Hasler, K., Fleckenstein, B., and Schaffner, W. (1985). A very strong enhancer is located upstream of an immediate early gene of human cytomegalovirus. Cell 41, 521-530. doi: 10.1016/S0092-8674(85)80025-8

Buehler, J., Carpenter, E., Zeltzer, S., Igarashi, S., Rak, M., Mikell, I., et al. (2019). Host signaling and EGR1 transcriptional control of human cytomegalovirus replication and latency. PLoS Pathog 15:e1008037. doi: 10.1371/journal.ppat.1008037

Buehler, J., Zeltzer, S., Reitsma, J., Petrucelli, A., Umashankar, M., Rak, M., et al. (2016). Opposing regulation of the EGF receptor: a molecular switch controlling cytomegalovirus latency and replication. PLoS Pathog 12:e1005655. doi: 10.1371/journal.ppat.1005655

Businger, R., Deutschmann, J., Gruska, I., Milbradt, J., Wiebusch, L., Gramberg, T., et al. (2019). Human cytomegalovirus overcomes SAMHD1 restriction in macrophages via pUL97. Nat. Microbiol. 4, 2260-2272. doi: 10.1038/s41564-019-0557-8

Caviness, K., Bughio, F., Crawford, L. B., Streblow, D. N., Nelson, J. A., Caposio, P., et al. (2016). Complex interplay of the UL136 isoforms balances cytomegalovirus replication and latency. MBio 7:e01986. doi: 10.1128/mBio.01986-15

Caviness, K., Cicchini, L., Rak, M., Umashankar, M., and Goodrum, F. (2014). Complex expression of the UL136 gene of human cytomegalovirus results in multiple protein isoforms with unique roles in replication. J. Virol. 88, 14412-14425. doi: 10.1128/JVI.02711-14

Chaturvedi, S., Klein, J., Vardi, N., Bolovan-Fritts, C., Wolf, M., Du, K., et al. (2020). A molecular mechanism for probabilistic bet hedging and its role in viral latency. Proc. Natl. Acad. Sci. U.S.A. 117, 17240-17248. doi: 10.1073/pnas.1914430117

Cheng, S., Caviness, K., Buehler, J., Smithey, M., Nikolich-Zugich, J., and Goodrum, F. (2017). Transcriptome-wide characterization of human cytomegalovirus in natural infection and experimental latency. Proc. Natl. Acad. Sci. U.S.A. 114, E10586-E10595. doi: 10.1073/pnas.1710522114 contributed to figure. All authors contributed to the article and approved the submitted version.

\section{FUNDING}

This work was supported by NIH grants AI143191 to FG, NM, and JK and AI127335 to FG and AI123811 and AI103311 to NM. DC-M is supported by a fellowship from the American Heart Association.
Cherrington, J. M., Khoury, E. L., and Mocarski, E. S. (1991). Human cytomegalovirus ie2 negatively regulates alpha gene expression via a short target sequence near the transcription start site. J. Virol. 65, 887-896. doi: 10.1128/JVI.65.2.887-896.1991

Cliffe, A. R., Coen, D. M., and Knipe, D. M. (2013). Kinetics of facultative heterochromatin and polycomb group protein association with the herpes simplex viral genome during establishment of latent infection. MBio 4, e00590-12. doi: 10.1128/mBio.00590-12

Cliffe, A. R., Garber, D. A., and Knipe, D. M. (2009). Transcription of the herpes simplex virus latency-associated transcript promotes the formation of facultative heterochromatin on lytic promoters. J. Virol. 83, 8182-8190. doi: 10.1128/JVI.00712-09

Cliffe, A. R., and Wilson, A. C. (2017). Restarting lytic gene transcription at the onset of herpes simplex virus reactivation. J. Virol. 91:e02621-18. doi: 10.1128/JVI.01419-16

Collins-McMillen, D., Buehler, J., Peppenelli, M., and Goodrum, F. (2018). Molecular determinants and the regulation of human cytomegalovirus latency and reactivation. Viruses 10:444. doi: 10.3390/v10080444

Collins-McMillen, D., and Goodrum, F.D. (2017). The loss of binary: Pushing the herpesvirus latency paradigm. Curr. Clin. Microbiol. Rep. 4, 124-131. doi: $10.1007 / \mathrm{s} 40588-017-0072-8$.

Collins-McMillen, D., Rak, M., Buehler, J. C., Igarashi-Hayes, S., Kamil, J. P., Moorman, N. J., et al. (2019). Alternative promoters drive human cytomegalovirus reactivation from latency. Proc. Natl. Acad. Sci. U.S.A. 116, 17492-17497. doi: 10.1073/pnas.1900783116

Crawford, L. B., Kim, J. H., Collins-McMillen, D., Lee, B. J., Landais, I., Held, C., et al. (2018). Human cytomegalovirus encodes a novel FLT3 receptor ligand necessary for hematopoietic cell differentiation and viral reactivation. MBio 9, e00682-18. doi: 10.1128/mBio.00682-18

Dag, F., Dolken, L., Holzki, J., Drabig, A., Weingartner, A., Schwerk, J., et al. (2014). Reversible silencing of cytomegalovirus genomes by type I interferon governs virus latency. PLoS Pathog 10:e1003962. doi: 10.1371/journal.ppat.1003962

Davison, A. J., Dolan, A., Akter, P., Addison, C., Dargan, D. J., Alcendor, D. J., et al. (2003). The human cytomegalovirus genome revisited: comparison with the chimpanzee cytomegalovirus genome. J. Gen. Virol. 84 (Pt. 1), 17-28. doi: 10.1099/vir.0.18606-0

Davuluri, R. V., Suzuki, Y., Sugano, S., Plass, C., and Huang, T. H. (2008). The functional consequences of alternative promoter use in mammalian genomes. Trends Genet. 24, 167-177. doi: 10.1016/j.tig.2008.01.008

Deutschmann, J., Schneider, A., Gruska, I., Vetter, B., Thomas, D., Kiessling, M., et al. (2019). A viral kinase counteracts in vivo restriction of murine cytomegalovirus by SAMHD1. Nat. Microbiol. 4, 2273-2284. doi: 10.1038/s41564-019-0529-z

Dupont, L., Du, L., Poulter, M., Choi, S., McIntosh, M., and Reeves, M. B. (2019). Src family kinase activity drives cytomegalovirus reactivation by recruiting MOZ histone acetyltransferase activity to the viral promoter. J. Biol. Chem. 294, 12901-12910. doi: 10.1074/jbc.RA119.009667

Elder, E. G., Krishna, B. A., Williamson, J., Lim, E. Y., Poole, E., Sedikides, G. X., et al. (2019). Interferon-responsive genes are targeted during the establishment of human cytomegalovirus latency. MBio 10. doi: 10.1128/mBio. 02574-19

Forte, E., Swaminathan, S., Schroeder, M. W., Kim, J. Y., Terhune, S. S., and Hummel, M. (2018). Tumor necrosis factor alpha induces reactivation of human cytomegalovirus independently of myeloid cell differentiation 
following posttranscriptional establishment of latency. MBio 9, e01560-18. doi: $10.1128 / \mathrm{mBio} .01560-18$

Furman, D., Jojic, V., Sharma, S., Shen-Orr, S. S., Angel, C. J., OnengutGumuscu, S., et al. (2015). Cytomegalovirus infection enhances the immune response to influenza. Sci. Transl. Med. 7:281ra243. doi: 10.1126/scitranslmed. aaa2293

Goodrum, F. (2016). Human cytomegalovirus latency: approaching the gordian knot. Annu Rev Virol. 3, 333-357. doi: 10.1146/annurev-virology-110615-042422

Grainger, L., Cicchini, L., Rak, M., Petrucelli, A., Fitzgerald, K. D., Semler, B. L., et al. (2010). Stress-inducible alternative translation initiation of human cytomegalovirus latency protein pUL138. J. Virol. 84, 9472-9486. doi: 10.1128/JVI.00855-10

Groves, I. J., Reeves, M. B., and Sinclair, J. H. (2009). Lytic infection of permissive cells with human cytomegalovirus is regulated by an intrinsic 'preimmediate-early' repression of viral gene expression mediated by histone posttranslational modification. J. Gen. Virol. 90 (Pt. 10), 2364-2374. doi: 10.1099/ vir.0.012526-0

Groves, I. J., and Sinclair, J. H. (2007). Knockdown of hDaxx in normally nonpermissive undifferentiated cells does not permit human cytomegalovirus immediate-early gene expression. J. Gen. Virol. 88 (Pt. 11), 2935-2940. doi: 10.1099/vir.0.83019-0

Hale, A. E., Collins-McMillen, D., Lenarcic, E. M., Igarashi, S., Kamil, J. P., Goodrum, F., et al. (2020). FOXO transcription factors activate alternative major immediate early promoters to induce human cytomegalovirus reactivation. Proc. Natl. Acad. Sci. U.S.A. 117, 18764-18770. doi: $10.1073 /$ pnas. 2002651117

Hopcraft, S. E., Pattenden, S. G., James, L. I., Frye, S., Dittmer, D. P., and Damania, B. (2018). Chromatin remodeling controls kaposi's sarcomaassociated herpesvirus reactivation from latency. PLoS Pathog 14:e1007267. doi: 10.1371/journal.ppat.1007267

Huang, M. M., Kew, V. G., Jestice, K., Wills, M. R., and Reeves, M. B. (2012). Efficient human cytomegalovirus reactivation is maturation dependent in the langerhans dendritic cell lineage and can be studied using a CD14+ experimental latency model. J. Virol. 86, 8507-8515. doi: 10.1128/JVI. 00598-12

Huang, T. H., Oka, T., Asai, T., Okada, T., Merrills, B. W., Gertson, P. N., et al. (1996). Repression by a differentiation-specific factor of the human cytomegalovirus enhancer. Nucleic Acids Res. 24, 1695-1701. doi: 10.1093/nar/24.9.1695

Ibanez, C. E., Schrier, R., Ghazal, P., Wiley, C., and Nelson, J. A. (1991). Human cytomegalovirus productively infects primary differentiated macrophages. J. Virol. 65, 6581-6588. doi: 10.1128/JVI.65.12.6581-6588. 1991

Jarvis, M. A., and Nelson, J. A. (2002). Human cytomegalovirus persistence and latency in endothelial cells and macrophages. Curr. Opin. Microbiol. 5, 403-407. doi: 10.1016/S1369-5274(02)00334-X

Jarvis, M. A., and Nelson, J. A. (2007). Human cytomegalovirus tropism for endothelial cells: not all endothelial cells are created equal. J. Virol. 81, 2095-2101. doi: 10.1128/JVI.01422-06

Kew, V. G., Yuan, J., Meier, J., and Reeves, M. B. (2014). Mitogen and stress activated kinases act co-operatively with CREB during the induction of human cytomegalovirus immediate-early gene expression from latency. PLoS Pathog 10:e1004195. doi: 10.1371/journal.ppat. 1004195

Kim, E. T., Roche, K. L., Kulej, K., Spruce, L. A., Seeholzer, S. H., Coen, D. M., et al. (2019). SAMHD1 Modulates early steps during human cytomegalovirus infection by limiting NF-кB activation. Cell Rep. 28, 434-448.e436. doi: 10.1016/j.celrep.2019. 06.027

Kim, J. H., Collins-McMillen, D., Buehler, J. C., Goodrum, F. D., and Yurochko, A. D. (2017). Human cytomegalovirus requires epidermal growth factor receptor signaling to enter and initiate the early steps in the establishment of latency in CD34(+) human progenitor cells. J. Virol. 91, e01206-e01216. doi: 10.1128/JVI.01206-16

Krishna, B. A., Humby, M. S., Miller, W. E., and O'Connor, C. M. (2019). Human cytomegalovirus G protein-coupled receptor US28 promotes latency by attenuating c-fos. Proc. Natl. Acad. Sci. U.S.A. 116, 1755-1764 doi: $10.1073 /$ pnas. 1816933116

Krishna, B. A., Wass, A. B., and O'connor, C. (2020). Activator protein-1 of the major immediate early locus is a determinant of cytomegalovirus reactivation from latency. Proc. Natl. Acad. Sci. USA. (2020). doi: 10.1073/pnas.2009420117. [Epub ahead of print].

Liang, R., Rimmele, P., Bigarella, C. L., Yalcin, S., and Ghaffari, S. (2016). Evidence for AKT-independent regulation of FOXO1 and FOXO3 in haematopoietic stem and progenitor cells. Cell Cycle 15, 861-867. doi: 10.1080/15384101.2015.1123355

Lieberman, P. M. (2015). Chromatin structure of epstein-barr virus latent episomes. Curr. Top Microbiol. Immunol. 390 (Pt2. 1), 71-102. doi: 10.1007/978-3-319-22822-8_5

Lin, Y. T., Prendergast, J., and Grey, F. (2017). The host ubiquitin-dependent segregase $\mathrm{VCP} / \mathrm{p} 97$ is required for the onset of human cytomegalovirus replication. PLoS Pathog 13:e1006329. doi: 10.1371/journal.ppat.1006329

Liu, B., Hermiston, T. W., and Stinski, M. F. (1991). A cis-acting element in the major immediate early (IE) promoter of human cytomegalovirus is required for negative regulation by IE2. J. Virol. 65, 897-903. doi: 10.1128/JVI.65.2.897-903.1991

Liu, B., and Stinski, M. F. (1992). Human cytomegalovirus contains a tegument protein that enhances transcription from promoters with upstream ATF and AP-1 cis-acting elements. J. Virol. 66, 4434-4444. doi: 10.1128/JVI.66.7.4434-4444.1992

Liu, X., Yuan, J., Wu, A. W., McGonagill, P. W., Galle, C. S., and Meier, J. L. (2010). Phorbol ester-induced human cytomegalovirus major immediate-early (MIE) enhancer activation through PKC-delta, CREB, and NF- $\kappa$ B desilences MIE gene expression in quiescently infected human pluripotent NTera2 cells. J. Virol. 84, 8495-8508. doi: 10.1128/JVI.00416-10

Liu, X. F., Yan, S., Abecassis, M., and Hummel, M. (2008). Establishment of murine cytomegalovirus latency in vivo is associated with changes in histone modifications and recruitment of transcriptional repressors to the major immediate-early promoter. J. Virol. 82, 10922-10931. doi: 10.1128/JVI.00865-08

Lubon, H., Ghazal, P., Hennighausen, L., Reynolds-Kohler, C., Lockshin, C., and Nelson, J. (1989). Cell-specific activity of the modulator region in the human cytomegalovirus major immediate-early gene. Mol. Cell. Biol. 9, 1342-1345. doi: 10.1128/MCB.9.3.1342

Macias, M. P., and Stinski, M. F. (1993). An in vitro system for human cytomegalovirus immediate early 2 protein (IE2)-mediated site-dependent repression of transcription and direct binding of IE2 to the major immediate early promoter. Proc. Natl. Acad. Sci. U.S.A. 90, 707-711. doi: 10.1073/pnas.90.2.707

Martinez, F. P., Cruz, R., Lu, F., Plasschaert, R., Deng, Z., Rivera-Molina, Y. A., et al. (2014). CTCF binding to the first intron of the major immediate early (MIE) gene of human cytomegalovirus (HCMV) negatively regulates MIE gene expression and HCMV replication. J. Virol. 88, 7389-7401. doi: 10.1128/JVI.00845-14

Mason, R., Groves, I. J., Wills, M. R., Sinclair, J. H., and Reeves, M. B. (2020). Human cytomegalovirus major immediate early transcripts arise predominantly from the canonical major immediate early promoter in reactivating progenitor-derived dendritic cells. J. Gen. Virol. 101, 635-644. doi: 10.1099/jgv.0.001419

Merkl, P. E., and Knipe, D. M. (2019). Role for a filamentous nuclear assembly of IFI16, DNA, and host factors in restriction of herpesviral infection. MBio 10:e2621-18 doi: 10.1128/mBio.02621-18

Mikell, I., Crawford, L. B., Hancock, M. H., Mitchell, J., Buehler, J., Goodrum, F., et al. (2019). HCMV miR-US22 down-regulation of EGR-1 regulates CD34+ hematopoietic progenitor cell proliferation and viral reactivation. PLoS Pathog 15:e1007854. doi: 10.1371/journal.ppat.1007854

Mizrahi, O., Nachshon, A., Shitrit, A., Gelbart, I. A., Dobesova, M., Brenner, S., et al. (2018). Virus-induced changes in mRNA secondary structure uncover cis-regulatory elements that directly control gene expression. Mol. Cell 72, 862-874.e865. doi: 10.1016/j.molcel.2018.09.003

Murphy, E., Rigoutsos, I., Shibuya, T., and Shenk, T. E. (2003a). Reevaluation of human cytomegalovirus coding potential. Proc. Natl. Acad. Sci. U.S.A. 100, 13585-13590. doi: 10.1073/pnas.1735466100 
Murphy, E., Yu, D., Grimwood, J., Schmutz, J., Dickson, M., Jarvis, M. A., et al. (2003b). Coding potential of laboratory and clinical strains of human cytomegalovirus. Proc. Natl. Acad. Sci. U.S.A. 100, 14976-14981. doi: 10.1073/pnas.2136652100

Murphy, J. C., Fischle, W., Verdin, E., and Sinclair, J. H. (2002). Control of cytomegalovirus lytic gene expression by histone acetylation. EMBO J. 21, 1112-1120. doi: 10.1093/emboj/21.5.1112

Nelson, J. A., Reynolds-Kohler, C., and Smith, B. A. (1987). Negative and positive regulation by a short segment in the 5'-flanking region of the human cytomegalovirus major immediate-early gene. Mol. Cell. Biol. 7, 4125-4129. doi: 10.1128/MCB.7.11.4125

Nikolich-Zugich, J., Goodrum, F., Knox, K., and Smithey, M. J. (2017). Known unknowns: how might the persistent herpesvirome shape immunity and aging? Curr. Opin. Immunol. 48, 23-30. doi: 10.1016/j.coi.2017.07.011

Nonkwelo, C., Skinner, J., Bell, A., Rickinson, A., and Sample, J. (1996). Transcription start sites downstream of the epstein-barr virus (EBV) Fp promoter in early-passage burkitt lymphoma cells define a fourth promoter for expression of the EBV EBNA-1 protein. J. Virol. 70, 623-627. doi: 10.1128/JVI.70.1.623-627.1996

O'Connor, C. M., Nukui, M., Gurova, K. V., and Murphy, E. A. (2016). Inhibition of the FACT complex reduces transcription from the human cytomegalovirus major immediate early promoter in models of lytic and latent replication. J. Virol. 90, 4249-4253. doi: 10.1128/JVI.02501-15

Oduro, J. D., Uecker, R., Hagemeier, C., and Wiebusch, L. (2012). Inhibition of human cytomegalovirus immediate-early gene expression by cyclin A2-dependent kinase activity. J. Virol. 86, 9369-9383. doi: 10.1128/JVI. 07181-11

Orzalli, M. H., DeLuca, N. A., and Knipe, D. M. (2012). Nuclear IFI16 induction of IRF-3 signaling during herpesviral infection and degradation of IFI16 by the viral ICP0 protein. Proc. Natl. Acad. Sci. U.S.A. 109, E3008-3017. doi: 10.1073/pnas.1211302109

Paik, J. H., Kollipara, R., Chu, G., Ji, H., Xiao, Y., Ding, Z., et al. (2007). FoxOs are lineage-restricted redundant tumor suppressors and regulate endothelial cell homeostasis. Cell 128, 309-323. doi: 10.1016/j.cell.2006.12.029

Pan, C., Zhu, D., Wang, Y., Li, L., Li, D., Liu, F., et al. (2016). Human cytomegalovirus miR-UL148D facilitates latent viral infection by targeting host cell immediate early response gene 5. PLoS Pathog 12:e1006007. doi: 10.1371/journal.ppat.1006007

Parida, M., Nilson, K. A., Li, M., Ball, C. B., Fuchs, H. A., Lawson, C. K., et al. (2019). Nucleotide resolution comparison of transcription of human cytomegalovirus and host genomes reveals universal use of RNA polymerase II elongation control driven by dissimilar core promoter elements. MBio 10, e02047-18. doi: 10.1128/mBio.02047-18

Pizzorno, M. C., and Hayward, G. S. (1990). The IE2 gene products of human cytomegalovirus specifically down-regulate expression from the major immediate-early promoter through a target located near the cap site. J. Virol. 64, 6154-6165. doi: 10.1128/JVI.64.12.6154-6165.1990

Rak, M. A., Buehler, J., Zeltzer, S., Reitsma, J., Molina, B., Terhune, S., et al. (2018). Human cytomegalovirus UL135 interacts with host adaptor proteins to regulate epidermal growth factor receptor and reactivation from latency. $J$. Virol. 92:e00919-18. doi: 10.1128/JVI.00919-18

Rauwel, B., Jang, S. M., Cassano, M., Kapopoulou, A., Barde, I., and Trono, D. (2015). Release of human cytomegalovirus from latency by a KAP1/TRIM28 phosphorylation switch. Elife 4:e06068. doi: 10.7554/eLife.06068.035

Reeves, M., Murphy, J., Greaves, R., Fairley, J., Brehm, A., and Sinclair, J. (2006). Autorepression of the human cytomegalovirus major immediate-early promoter/enhancer at late times of infection is mediated by the recruitment of chromatin remodeling enzymes by IE86. J. Virol. 80, 9998-10009. doi: 10.1128/JVI.01297-06

Reeves, M., and Sinclair, J. (2013). Regulation of human cytomegalovirus transcription in latency: beyond the major immediate-early promoter. Viruses 5, 1395-1413. doi: 10.3390/v5061395

Reeves, M. B., and Compton, T. (2011). Inhibition of inflammatory interleukin6 activity via extracellular signal-regulated kinase-mitogen-activated protein kinase signaling antagonizes human cytomegalovirus reactivation from dendritic cells. J. Virol. 85, 12750-12758. doi: 10.1128/JVI.05878-11

Reeves, M. B., MacAry, P. A., Lehner, P. J., Sissons, J. G., and Sinclair, J. H. (2005). Latency, chromatin remodeling, and reactivation of human cytomegalovirus in the dendritic cells of healthy carriers. Proc. Natl. Acad. Sci. U.S.A. 102, 4140-4145. doi: 10.1073/pnas.0408994102

Saffert, R. T., and Kalejta, R. F. (2006). Inactivating a cellular intrinsic immune defense mediated by daxx is the mechanism through which the human cytomegalovirus pp71 protein stimulates viral immediate-early gene expression. J. Virol. 80, 3863-3871. doi: 10.1128/JVI.80.8.3863-3871.2006

Saffert, R. T., and Kalejta, R. F. (2007). Human cytomegalovirus gene expression is silenced by daxx-mediated intrinsic immune defense in model latent infections established in vitro. J. Virol. 81, 9109-9120. doi: 10.1128/JVI.00827-07

Saffert, R. T., Penkert, R. R., and Kalejta, R. F. (2010). Cellular and viral control over the initial events of human cytomegalovirus experimental latency in CD34+ cells. J. Virol. 84, 5594-5604. doi: 10.1128/JVI.00348-10

Sanchez, V., McElroy, A. K., Yen, J., Tamrakar, S., Clark, C. L., Schwartz, R. A., et al. (2004). Cyclin-dependent kinase activity is required at early times for accurate processing and accumulation of the human cytomegalovirus UL122-123 and UL37 immediate-early transcripts and at later times for virus production. $J$. Virol. 78, 11219-11232. doi: 10.1128/JVI.78.20.11219-11232.2004

Schaefer, B. C., Strominger, J. L., and Speck, S. H. (1995). Redefining the epsteinbarr virus-encoded nuclear antigen EBNA-1 gene promoter and transcription initiation site in group I burkitt lymphoma cell lines. Proc. Natl. Acad. Sci. U.S.A. 92, 10565-10569. doi: 10.1073/pnas.92.23.10565

Shelbourn, S. L., Kothari, S. K., Sissons, J. G., and Sinclair, J. H. (1989). Repression of human cytomegalovirus gene expression associated with a novel immediate early regulatory region binding factor. Nucleic Acids Res. 17, 9165-9171. doi: 10.1093/nar/17.22.9165

Shnayder, M., Nachshon, A., Krishna, B., Poole, E., Boshkov, A., Binyamin, A., et al. (2018). Defining the transcriptional landscape during cytomegalovirus latency with single-cell rna sequencing. MBio 9, e00013-e00018. doi: $10.1128 / \mathrm{mBio} .00013-18$

Sinclair, J. H., Baillie, J., Bryant, L. A., Taylor-Wiedeman, J. A., and Sissons, J. G. (1992). Repression of human cytomegalovirus major immediate early gene expression in a monocytic cell line. J. Gen. Virol. 73 (Pt. 2), 433-435. doi: 10.1099/0022-1317-73-2-433

Singh, N., and Tscharke, D.C. (2020). Herpes simplex virus latency is noisier the closer we look. J. Virol. 94:e01701-19. doi: 10.1128/JVI.01701-19

Soderberg-Naucler, C., Fish, K. N., and Nelson, J. A. (1997). Reactivation of latent human cytomegalovirus by allogeneic stimulation of blood cells from healthy donors. Cell 91, 119-126. doi: 10.1016/S0092-8674(01)80014-3

Soderberg-Naucler, C., Streblow, D. N., Fish, K. N., Allan-Yorke, J., Smith, P. P., and Nelson, J. A. (2001). Reactivation of latent human cytomegalovirus in CD14(+) monocytes is differentiation dependent. J. Virol. 75, 7543-7554. doi: 10.1128/JVI.75.16.7543-7554.2001

Stenberg, R. M., Witte, P. R., and Stinski, M. F. (1985). Multiple spliced and unspliced transcripts from human cytomegalovirus immediate-early region 2 and evidence for a common initiation site within immediate-early region $1 . J$. Virol. 56, 665-675. doi: 10.1128/JVI.56.3.665-675.1985

Stern-Ginossar, N., Weisburd, B., Michalski, A., Le, V. T., Hein, M. Y., Huang, S. X., et al. (2012). Decoding human cytomegalovirus. Science 338, 1088-1093. doi: $10.1126 /$ science.1227919

Strassheim, S., Gennart, I., Muylkens, B., Andre, M., Rasschaert, D., and Laurent, S. (2016). Oncogenic Marek's disease herpesvirus encodes an isoform of the conserved regulatory immediate early protein ICP27 generated by alternative promoter usage. J. Gen. Virol. 97, 2399-2410. doi: 10.1099/jgv.0.000547

Streblow, D. N., Dumortier, J., Moses, A. V., Orloff, S. L., and Nelson, J. A. (2008). Mechanisms of cytomegalovirus-accelerated vascular disease: induction of paracrine factors that promote angiogenesis and wound healing. Curr. Top. Microbiol. Immunol. 325, 397-415. doi: 10.1007/978-3-540-77349-8_22

Taylor-Wiedeman, J., Sissons, P., and Sinclair, J. (1994). Induction of endogenous human cytomegalovirus gene expression after differentiation of monocytes from healthy carriers. J. Virol. 68, 1597-1604. doi: 10.1128/JVI.68.3.1597-1604.1994

Tempera, I., Klichinsky, M., and Lieberman, P. M. (2011). EBV latency types adopt alternative chromatin conformations. PLoS Pathog 7:e1002180. doi: 10.1371/journal.ppat.1002180

Teng, M. W., Bolovan-Fritts, C., Dar, R. D., Womack, A., Simpson, M. L., Shenk, T., et al. (2012). An endogenous accelerator for viral gene expression confers a fitness advantage. Cell 151, 1569-1580. doi: 10.1016/j.cell.2012. 11.051 
Thomsen, D. R., Stenberg, R. M., Goins, W. F., and Stinski, M. F. (1984). Promoter-regulatory region of the major immediate early gene of human cytomegalovirus. Proc. Natl. Acad. Sci. U.S.A. 81, 659-663. doi: 10.1073 /pnas.81.3.659

Tothova, Z., and Gilliland, D. G. (2007). FoxO transcription factors and stem cell homeostasis: insights from the hematopoietic system. Cell Stem Cell 1, 140-152. doi: 10.1016/j.stem.2007.07.017

Trojer, P., and Reinberg, D. (2007). Facultative heterochromatin: is there a distinctive molecular signature? Mol. Cell 28, 1-13. doi: 10.1016/j.molcel.2007.09.011

Umashankar, M., Petrucelli, A., Cicchini, L., Caposio, P., Kreklywich, C. N., Rak, M., et al. (2011). A novel human cytomegalovirus locus modulates cell type-specific outcomes of infection. PLoS Pathog 7:e1002444. doi: 10.1371 /journal.ppat.1002444

Umashankar, M., Rak, M., Bughio, F., Zagallo, P., Caviness, K., and Goodrum, F. D. (2014). Antagonistic determinants controlling replicative and latent states of human cytomegalovirus infection. J. Virol. 88, 5987-6002. doi: 10.1128/JVI.03506-13

Wagenknecht, N., Reuter, N., Scherer, M., Reichel, A., Muller, R., and Stamminger, T. (2015). Contribution of the major ND10 proteins PML, hDaxx and Sp100 to the regulation of human cytomegalovirus latency and lytic replication in the monocytic cell line THP-1. Viruses 7, 2884-2907. doi: 10.3390/v7062751

Wakeman, B. S., Izumiya, Y., and Speck, S. H. (2017). Identification of novel kaposi's sarcoma-associated herpesvirus Orf50 transcripts: discovery of new RTA isoforms with variable transactivation potential. J. Virol 91:e01434-16. doi: 10.1128/JVI.01434-16

Woisetschlaeger, M., Yandava, C. N., Furmanski, L. A., Strominger, J. L., and Speck, S. H. (1990). Promoter switching in Epstein-Barr virus during the initial stages of infection of B lymphocytes. Proc. Natl. Acad. Sci. U.S.A. 87, 1725-1729. doi: $10.1073 /$ pnas.87.5.1725
Woodhall, D. L., Groves, I. J., Reeves, M. B., Wilkinson, G., and Sinclair, J. H. (2006). Human Daxx-mediated repression of human cytomegalovirus gene expression correlates with a repressive chromatin structure around the major immediate early promoter. J. Biol. Chem. 281, 37652-37660. doi: 10.1074/jbc.M604273200

Wright, E., Bain, M., Teague, L., Murphy, J., and Sinclair, J. (2005). Ets-2 repressor factor recruits histone deacetylase to silence human cytomegalovirus immediate-early gene expression in non-permissive cells. J. Gen. Virol. 86 (Pt. 3), 535-544. doi: 10.1099/vir.0.80352-0

Yalcin, S., Zhang, X., Luciano, J. P., Mungamuri, S. K., Marinkovic, D., Vercherat, C., et al. (2008). Foxo3 is essential for the regulation of ataxia telangiectasia mutated and oxidative stress-mediated homeostasis of hematopoietic stem cells. J. Biol. Chem. 283, 25692-25705. doi: 10.1074/jbc.M800517200

Yuan, J., Li, M., Torres, Y. R., Galle, C. S., and Meier, J. L. (2015). Differentiation-coupled Induction of human cytomegalovirus replication by union of the major enhancer retinoic acid, cyclic AMP, and NF$\kappa \mathrm{B}$ response elements. J. Virol. 89, 12284-12298. doi: 10.1128/JVI. 00965-15

Conflict of Interest: The authors declare that the research was conducted in the absence of any commercial or financial relationships that could be construed as a potential conflict of interest.

Copyright (c) 2020 Collins-McMillen, Kamil, Moorman and Goodrum. This is an open-access article distributed under the terms of the Creative Commons Attribution License (CC BY). The use, distribution or reproduction in other forums is permitted, provided the original author(s) and the copyright owner(s) are credited and that the original publication in this journal is cited, in accordance with accepted academic practice. No use, distribution or reproduction is permitted which does not comply with these terms. 